\title{
Effect of DC Magnetic Field on Crystal Orientation and Mechanical Properties of Remelting Mg-Y-Cu-Zr Alloy
}

\author{
Man-Qing HUANG ${ }^{1, a}$, Quan ZHOU ${ }^{1, b}$, Feng LIU ${ }^{1, c}$, Lei ZHANG ${ }^{1, d}$ \\ ${ }^{1}$ School of Aeronautical Manufacturing Engineering, Nanchang Hangkong University, Nanchang \\ 330063, China \\ a780235327@qq.com, blgclzq@sina.com, ‘770400705@qq.com, dniatzhanglei01@126.com
}

Keywords: DC magnetic field, Mg-Y-Cu-Zr alloy, crystal orientation, mechanical properties, long period ordered structure

\begin{abstract}
The influence of different strength (0-1.4T) of DC magnetic field on crystal orientation and mechanical properties of remelting $\mathrm{Mg}-\mathrm{Y}-\mathrm{Cu}-\mathrm{Zr}$ alloy reinforced by long period ordered structure were studied. The results show that with the increase of strength of magnetic field, primary phase and second phase of the alloy decrease at first, and then coarsen. The turning point is $0.56 \mathrm{~T}$. The influence of DC magnetic field on crystal orientation of the alloy is not obvious when the strength of magnetic field is $0.28 \mathrm{~T}$. When the strength of DC magnetic field is more than $0.56 \mathrm{~T}$, the diffraction intensity of crystal plane (101) of $\alpha-\mathrm{Mg}$ matrix is weakened significantly, and the diffraction intensity of crystal planes (100) and (110) are enhanced. Mechanical properties of the alloy can be increased obviously with DC magnetic field treatment. When the strength of magnetic field is $1.4 \mathrm{~T}$, tensile strength and elongation of the alloy reach $237 \mathrm{MPa}$ and $8.5 \%$, which are increased by up to $38.6 \%$ and $33.4 \%$, respectively, compared with the alloy without treatment.
\end{abstract}

\section{Introduction}

Magnesium alloy, as a new type of constructional materials, offers numerous merits in physical, mechanical and casting properties, such as high specific strength and stiffness, good castability, good thermal and electric conductivity, which has been used widely in aeronautic, automotive, electronic industries field in recent years[1, 2]. $\mathrm{Mg}$ alloys reinforced by long-period ordered structure are promising candidates for lightweight structural materials developed recently. As a new kind of reinforced phase in $\mathrm{Mg}$ alloys, the long-period stacking order structure(LPSO) can improve mechanical properties of $\mathrm{Mg}$ alloy at room and high temperature greatly[3]. In 2006, Kawamura et al[4] found that $\mathrm{Mg}_{97} \mathrm{Y}_{2} \mathrm{Cu}_{1}$ cast alloy has an 18R-type long period ordered structure that forms coherently with the $\alpha-\mathrm{Mg}$ matrix during casting. Although mechanical properties of the alloy after deformation processing are excellent, mechanical properties of the alloy in cast still need to be improved.

DC magnetic field can improve solidification structure, crystal orientation and mechanical properties of the alloy, which has advantages of pollution-free and convenient operation. Zhong et al[5] found that the easy magnetization axe $\langle 310\rangle$ of the $\alpha$-Al grains was aligned parallel to the direction of the magnetic field leading to $\langle 310\rangle$ texture with the increment magnetic field intensity. Tan et al[6] found that when the strength of magnetic field was $1.4 \mathrm{~T}$, the (100) plane texture of pure magnesium was enhanced significantly and the elongation was increased by up to 544\% compared with the sample without DC magnetic field treatment. Li et al[7] found that the crystal plane (100) of $\mathrm{Cu}-10 \% \mathrm{Ag}$ alloy was enhanced significantly with $1 \mathrm{~T}$ horizontal magnetic field treatment. However, the effect of DC magnetic field on crystal orientation of magnesium alloy, especially for magnesium alloy reinforced by long-period ordered structure is less researched. In this work, the effects of DC magnetic field on crystal orientation and mechanical properties of $\mathrm{Mg}-\mathrm{Y}-\mathrm{Cu}$ alloy were studied, based on the addition of $1 \% \mathrm{Zr}$. 


\section{Experimental}

$\mathrm{Mg}-\mathrm{Y}-\mathrm{Cu}-\mathrm{Zr}$ alloy was employed as the raw material for this study. Its chemical compositions (wt, \%) are as followings: Y 6.84, $\mathrm{Cu} 2.46, \mathrm{Zr} 1$ and $\mathrm{Mg}$ balance. The experimental alloys were prepared from pure $\mathrm{Mg}, \mathrm{Mg}-30 \mathrm{Y}, \mathrm{Mg}-30 \mathrm{Cu}$ and $\mathrm{Mg}-30 \mathrm{Zr}$ master alloys. The alloy was first melted at $760{ }^{\circ} \mathrm{C}$ and held for 15 min under $\mathrm{CO}_{2}+\mathrm{SF}_{6}$ mixture protection in an electrical resistance furnace using a mild steel crucible, and then the melt was poured into a graphite mold with an inside diameter of $36 \mathrm{~mm}$, height of $70 \mathrm{~mm}$ and a wall-thickness of $3 \mathrm{~mm}$, which was preheated to the temperature of $180{ }^{\circ} \mathrm{C}$. The master alloys obtained from the above experiments were put into corundum crucible and remelted. The alloy melt was kept for 10 min at $740{ }^{\circ} \mathrm{C}$ after completely melted, and then quickly transferred to the DC magnetic field solidification device, placed in the central portion of the magnetic poles. Magnetic field was imposed to the melt until it was solidified completely. Different strength of magnetic field $(0 \mathrm{~T}$, $0.28 \mathrm{~T}, 0.56 \mathrm{~T}, 0.84 \mathrm{~T}, 1.12 \mathrm{~T}$ and $1.4 \mathrm{~T}$ ) was used in this experiment. Sketch map of DC magnetic field solidification device is showed in Figure 1, including power supplies, magnetic pole, corundum crucible, heat insulator, metal and firebrick. The strength of magnetic field was changed by adjusting the excitation voltage.

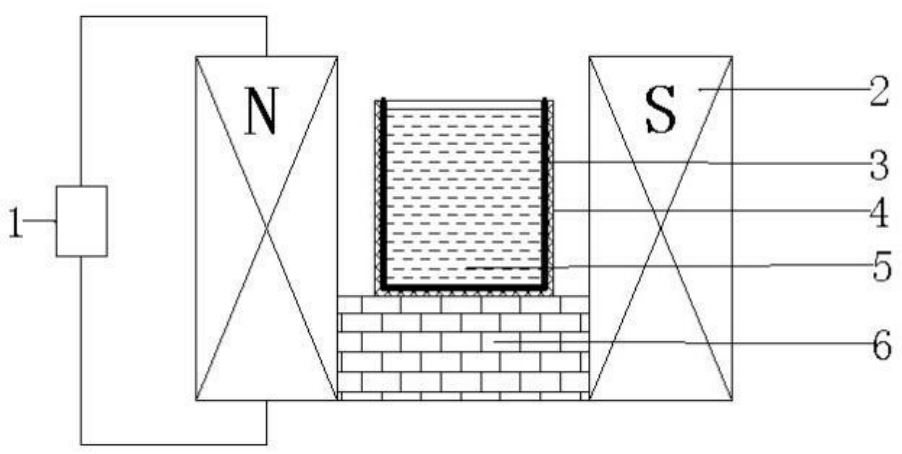

Fig.1 Sketch map of DC magnetic field solidification device. (1) Power supplies; (2) Magnetic pole; (3) Corundum crucible; (4) Heat insulator; (5) Metal; (6) Firebrick.

The specimens for microstructure observation were made from the transverse section of the $1 / 2$ height of the castings. After grinding and polishing, the specimens were etched with a solution of $0.5 \%$ (volume fraction) nitric acid and ethyl alcohol, and then examined by an optical microscope and Quanta200 type scanning electron microscope (SEM). Diffraction patterns of the sample were determined by X-ray diffractometer (XRD). Tensile specimens of magnesium alloys were prepared according to GB6397-86, and tested on WDW-200D type material testing machine.

\section{Results}

Figure 2 shows morphology of primary phase and second phase of $\mathrm{Mg}-\mathrm{Y}-\mathrm{Cu}-\mathrm{Zr}$ alloy under different strength of magnetic field. It can be seen that adding magnetic field or not, rosette-like primary crystals and continuous net-worked secondary phase can be normally observed in the alloy. When the strength of magnetic field changes from $0.28 \mathrm{~T}$ to $0.56 \mathrm{~T}$, primary grain of the alloy refines, and volume fraction of second phase increases. When the strength of magnetic field is at 0.84-1.4 T, with the increase of strength of magnetic field, primary grain of the alloy coarsens and the volume fraction of second phase decreases. The above results indicate that DC magnetic field has certain effect on solidification structure of the alloy, but not obviously.

Figure 3 shows the $\mathrm{X}$-ray diffraction (XRD) patterns of $\mathrm{Mg}-\mathrm{Y}-\mathrm{Cu}-\mathrm{Zr}$ alloy under different strength of magnetic field. It is shown that $\alpha-\mathrm{Mg}, \mathrm{Mg}_{12} \mathrm{Y}_{1} \mathrm{Cu}_{1}$ and $\mathrm{CuZr}_{2}$ phase in the alloy can be detected. Orientations of $\alpha-\mathrm{Mg}$ without magnetic field treatment are random. However, when strength of DC magnetic field is more than $0.28 \mathrm{~T}$, the diffraction intensity of crystal plane(101)of 
$\alpha-\mathrm{Mg}$ matrix is weakened, and crystal planes(100)and(110)are enhanced significantly.

The effects of different strength of magnetic field on mechanical properties of $\mathrm{Mg}-\mathrm{Y}-\mathrm{Cu}-\mathrm{Zr}$ alloy are shown in Fig.4. It is shown that with the increase of strength of magnetic field, the mechanical properties of the alloy increase firstly, and then decrease. When the strength of magnetic field is $0.56 \mathrm{~T}$, mechanical properties of the alloy are the best, tensile strength and elongation of the alloy reach $231 \mathrm{MPa}$ and $9.92 \%$, which are increased by up to $35.09 \%$ and $72.22 \%$, respectively, compared with the alloy without treatment.
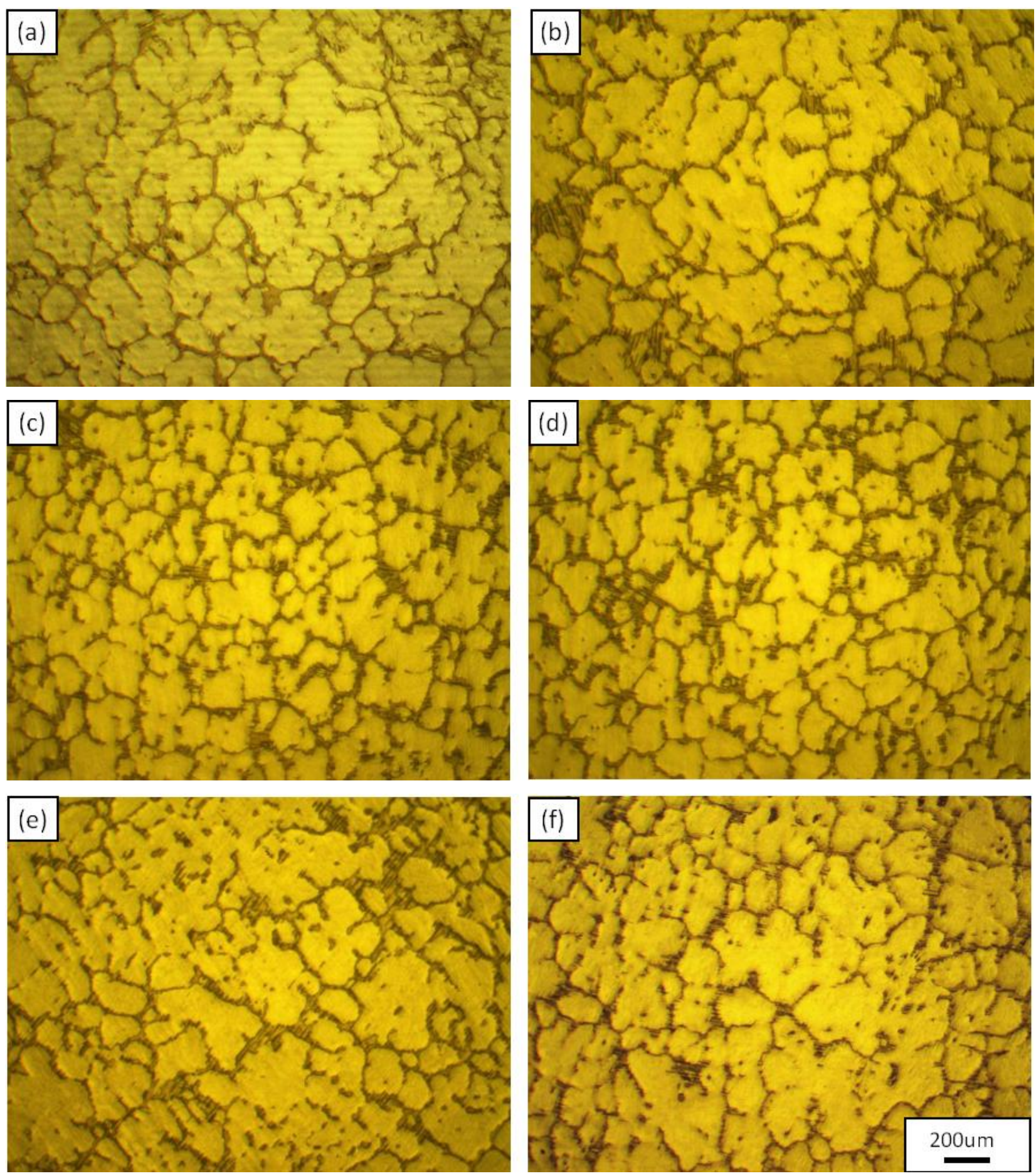

Fig.2 Effect of different strength of magnetic field on primary phase of $\mathrm{Mg}-\mathrm{Y}-\mathrm{Cu}-\mathrm{Zr}$ alloy.

$$
\text { (a)0 T; (b) } 0.28 \mathrm{~T} \text {; (c) } 0.56 \mathrm{~T} \text {; (d) } 0.84 \mathrm{~T} \text {; (e) } 1.12 \mathrm{~T} \text {; (f) } 1.4 \mathrm{~T}
$$


The fracture morphologies of the alloy without and with DC magnetic field treatment are shown in Fig.5. It is clear that without magnetic field treatment, tensile fracture of the alloy consists of cleavage plane and tearing ridge in different direction, which is quasi cleavage fracture. Fracture of the sample with magnetic treatment has obvious orientation, which is also quasi cleavage fracture. This is mainly because of the formation of preferred orientation under magnetic field.

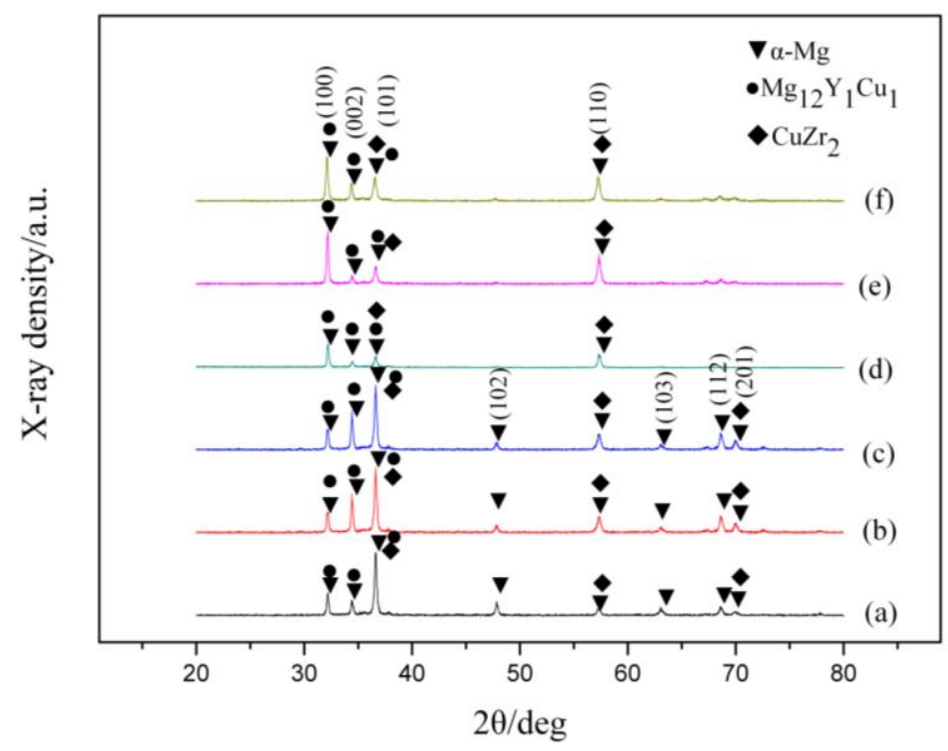

Fig.3 Effect of different strength of magnetic field on XRD patterns of $\mathrm{Mg}-\mathrm{Y}-\mathrm{Cu}-\mathrm{Zr}$ alloy. (a)0 T;(b) $0.28 \mathrm{~T}$;(c) $0.56 \mathrm{~T}$;(d) $0.84 \mathrm{~T}$;(e) $1.12 \mathrm{~T}$;(f) $1.4 \mathrm{~T}$

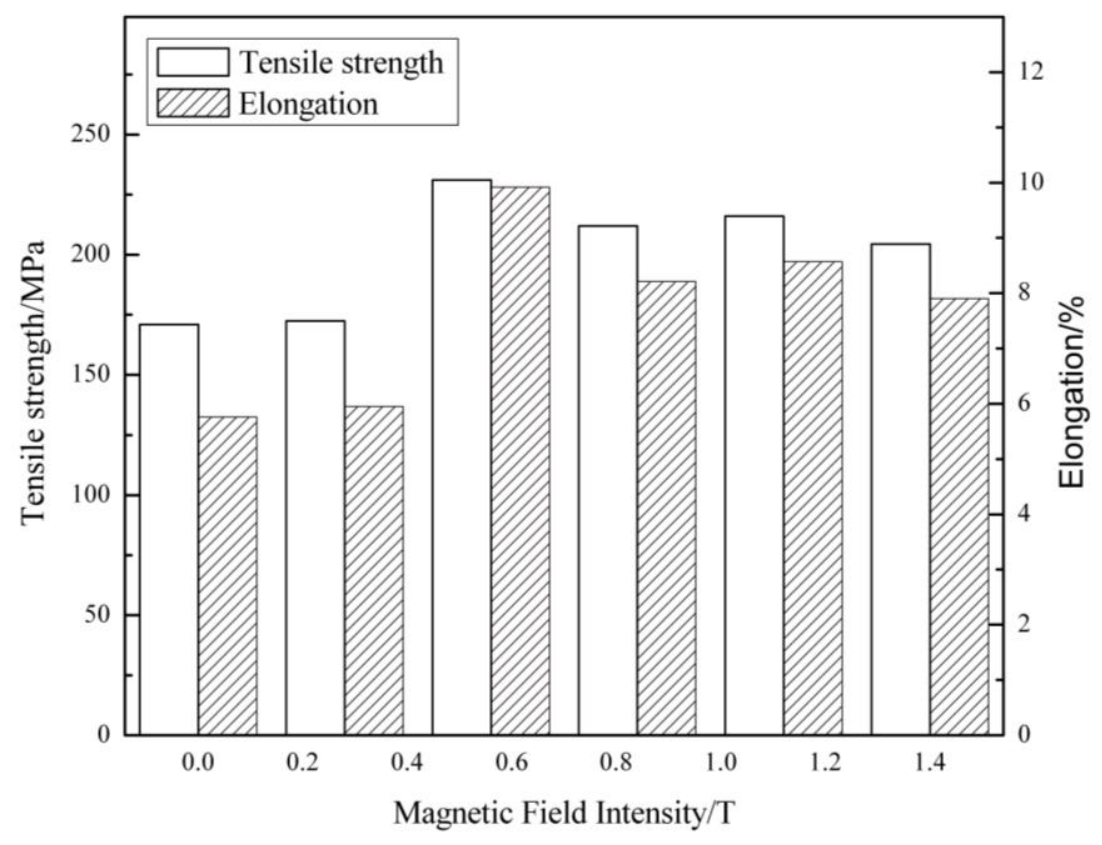

Fig.4 Effect of different strength of magnetic field on tensile strength and elongation of $\mathrm{Mg}-\mathrm{Y}-\mathrm{Cu}-\mathrm{Zr}$ alloy 

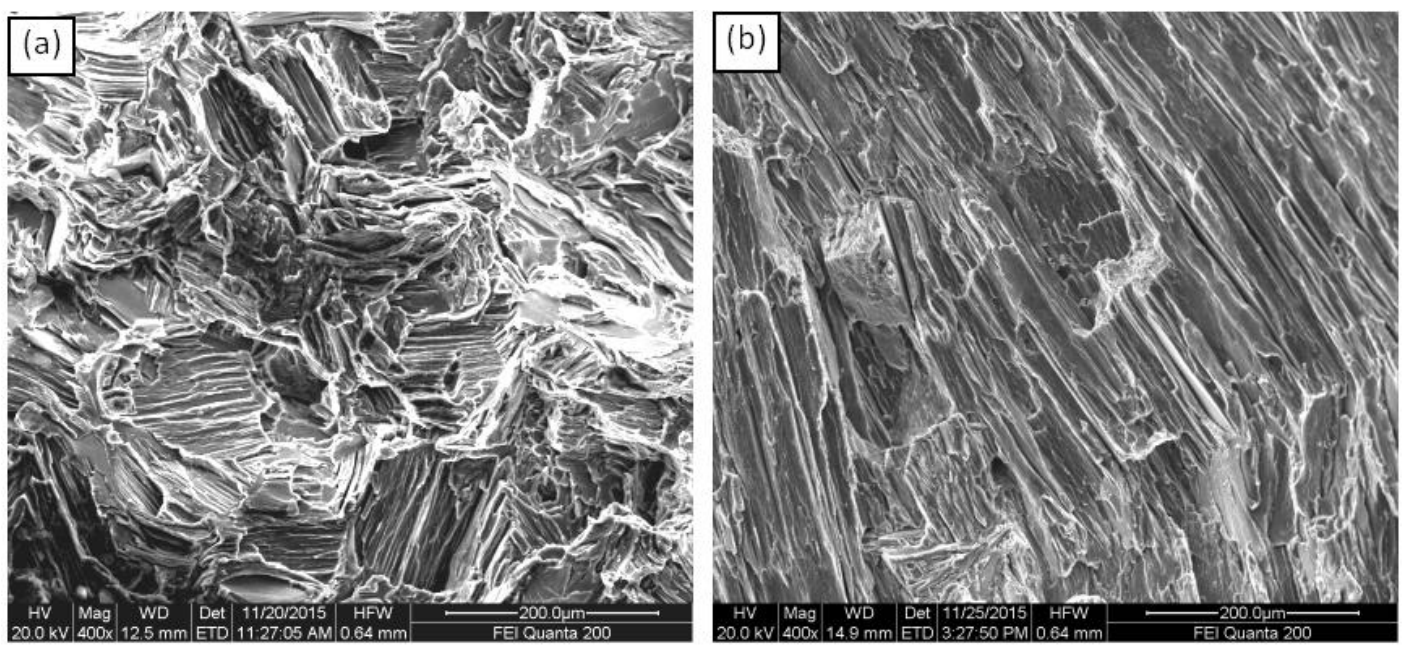

Fig.5 SEM fractographs of Mg-Y-Cu-Zr alloy

(a) without DC magnetic field treatment; (b) with DC magnetic field treatment

\section{Discussion}

The effect of magnetic field on electrical conductive melt has been studied for decades, and two main mechanisms have been recognized. On the one hand, the DC magnetic field can interact with the conductive fluid, resulting in an electromagnetic force $F_{M H D}[8]$ :

$$
F_{M H D}=-\sigma(\vec{u} \times \vec{B}) \times \vec{B}
$$

where, $\sigma$ is electrical conductivity, $\vec{u}$ is the liquid velocity field and $\vec{B}$ is the strength of magnetic field. The formula shows that the electromagnetic force $F_{M H D}$ can suppress natural convection of metal melt effectively, which leads to the increase of the barrier required to overcome during solidification, resulting in the decrease of nucleation rate. This phenomenon is called magnetic damping (MHD) effect.

On the other hand, due to the existence of thermoelectric effect, the thermal current will interact with the magnetic field, resulting in a thermoelectric magnetic force $F_{T E M C}$ [9]:

$$
F_{T E M C}=J_{T E} \times \vec{B}
$$

where, $J_{T E}$ is thermoelectric potential and $\vec{B}$ is the strength of magnetic field. Thermoelectric magnetic force $F_{T E M C}$ can strengthen heat and mass transfer during solidification of alloy, which can improve nucleation undercooling level and increase the nucleation rate of the alloy. This phenomenon is called thermoelectromagnetic convection (TEMC) effect.

When magnetic field is applied to the solidification course of $\mathrm{Mg}-\mathrm{Y}-\mathrm{Cu}-\mathrm{Zr}$ alloy, MHD and TEMC influence the melt at the same time, but they turn to a contest relationship and the solidified microstructure depends on the winner. As shown in the above formula, $F_{M H D}$ can be considered as a function of $B^{2}$ and $F_{T E M C}$ is directly proportional to $B$. The different increasing velocities between $F_{M H D}$ and $F_{T E M C}$ result in a difference $F$ because of their opposite directions when the magnetic field is applied. As shown in Fig. 2, when the strength of magnetic field is less than $0.56 \mathrm{~T}, F_{T E M C}$ is stronger than $F_{M H D}$ and difference $F$ increases with increasing the magnetic field intensity, which results in refinement of primary phase and second phase of the alloy. When the strength of magnetic field is $0.56 \mathrm{~T}$, the grain refinement effect of the alloy is the best. When the strength of magnetic field is more than $0.56 \mathrm{~T}, F_{M H D}$ is stronger than $F_{T E M C}$, which results in primary phase and second phase of the alloy coarsening. Grain refinement of the alloy is mainly influenced by $\mathrm{Zr}$, and less affected by DC magnetic field. Therefore, primary phase of $\mathrm{Mg}-\mathrm{Y}-\mathrm{Cu}-\mathrm{Zr}$ alloy does not change significantly under the DC magnetic field. In addition, earlier studies showed that DC magnetic field could restrain diffusion of solute elements in alloy [10]. The solubility of $\mathrm{Y}, \mathrm{Cu}$ inside grain of 
the alloy may be increased under magnetic field, which reduces the volume fraction of second phase in the alloy.

When Mg-Y-Cu-Zr alloy solidified under DC magnetic field, $\mathrm{Mg}$ crystal will be affected by magnetic force and the potential energy $U$ of the unit volume is given by the following equation[11].

$$
U=-\vec{M} \cdot \vec{B}, \quad M=\chi H
$$

here, $M$ is magnetization intensity, $B$ is magnetic flux density, $\chi$ is magnetic susceptibility and $H$ is intensity of the magnetic field. The formula shows that magnetic moment has a tendency to the outer magnetic field $\vec{B}$, so as to reduce the energy. The magnetic anisotropy crystal has different magnetization energy along the different crystal axis and the crystal is inclined to circumgyrate to the position where the energy is the lowest. Meanwhile, the crystallographic orientation is actually formed. As shown in Figure 4(c), the diffraction peak intensity of crystal planes (100) and (110) of $\alpha-\mathrm{Mg}$ matrix are much higher than that of other planes. It can be determined that the magnetic susceptibility of crystal planes (100) and (110) may be relatively high, resulting in formation of preferred orientation of the alloy.

Every crystal is magnetized as little magnets under DC magnetic field and these little magnets generate rotation tropism at the function of magnetic field force. The little magnets can generate interactivity with each other when part of the little magnets rotate to a definitive degree under magnetic fields and restrict each other in the migration and rotation process of primary $\alpha-\mathrm{Mg}$ crystals when the distance among the magnets reaches to a definitive useful range. Meanwhile, the migration and rotation process are also influenced by many factors, such as thermal agitation, which hinders the preferred orientation of the alloy. Therefore, only when the magnetic force is greater than the external resistance, the primary $\alpha-\mathrm{Mg}$ crystal can form preferred orientation on the crystal surface with high magnetic susceptibility. The influence of DC magnetic field on crystallization orientation of the alloy depends on the strength of magnetic field entirely. When the strength of magnetic field is $0.28 \mathrm{~T}$, the magnetic force is less than resistance of crystal rotation, therefore crystal orientation of the alloy has no obvious change. When the strength of magnetic field is more than $0.28 \mathrm{~T}$, the magnetic force is greater than the external resistance of crystal rotation, so significant preferred orientation forms in the alloy.

Magnesium alloy is polycrystalline materials, which can be regarded as a collection of small grain, its mechanical properties depend on grain size, morphology of second phase and crystal orientation. In this experiments, the mechanical properties of $\mathrm{Mg}-\mathrm{Y}-\mathrm{Cu}-\mathrm{Zr}$ alloy should be mainly affected by solidified structure and crystal orientation.

Plastic deformation capacity and mechanical properties of magnesium alloy are closely related to the crystal orientation of the alloy due to the anisotropy of magnesium grains. The relationship between them is given by the following equation[12]:

$$
\tau=\mu \sigma
$$

here, $\mu$ is Schmid factor, $\tau$ is critical resolved shear stress and $\sigma$ is strength of polycrystalline materials. The formula shows that the slip system is easy to start when the alloy is stretched to the crystal plane with larger Schmid factor. Studies found that formation of preferred orientation can alter the Schmid factors of all slips of magnesium alloy, especially the basal slip system $\{0001\}[11 \overline{2} 0]$, which will result in more slip mode being activated, thus affecting the mechanical properties of magnesium alloys[13].

When the strength of magnetic field is $0.28 \mathrm{~T}$, solidified structure and crystal orientation of the alloy have no obvious change, and thus the mechanical properties of the alloy are in accordance with the alloy without treatment. When the strength of magnetic field is greater than $0.28 \mathrm{~T}$, the obvious preferred orientation of the alloy is formed on the crystal planes (100) and (110), which may induce texture strengthening and improve the mechanical properties of the alloy. When the strength of magnetic field is $0.56 \mathrm{~T}$, because of microstructure refinement and formation of 
preferred orientation, mechanical properties of the alloy are the best. When the strength of magnetic field is at 0.56-1.4 $\mathrm{T}$, because of grain coarsening, mechanical properties of the alloy decrease slightly. But this difference is not noticeable because of the formation of preferential orientation.

\section{Conclusions}

1) With the increase of strength of magnetic field, primary phase and second phase of the alloy decrease at first, and then coarsen. The turning point is $0.56 \mathrm{~T}$.

2) Preferred orientation of the alloy is enhanced significantly when the strength of DC magnetic field is more than $0.28 \mathrm{~T}$. The diffraction intensity of crystal plane (101) of $\alpha-\mathrm{Mg}$ matrix is weakened, and crystal planes (100) and (110) are enhanced significantly with the increase of strength of magnetic field.

3) Mechanical properties of the alloy are increased obviously with DC magnetic field treatment. When the strength of magnetic field is $0.56 \mathrm{~T}$, mechanical properties of the alloy are the best, the tensile strength and elongation of the alloy reach $231 \mathrm{MPa}$ and $9.92 \%$, which are increased by up to $35.09 \%$ and $72.22 \%$, respectively, compared with the alloy without treatment.

\section{Acknowledgements}

This work was financially supported by the National Natural Science Foundation Project (51261026 and 51401102).

\section{References}

[1] K.Hazeli, A.Sadeghi, M.O.Pekguleryuz and A.Kontsos: Materials Science and Engineering A Vol. 578(2013), p.383-393

[2] P.S.Roodposhti, A.Sarkar and K.L.Murty: Materials Science and Engineering A Vol. 626(2015), p.195-202

[3] Y.Kawamura, K.Hayashi and A.Inoue: Mater Trans JIM Vol.42(2001), p.1171

[4] Y. Kawamura, T. Morisaka and S. Izumi: Scripta Mater. Vol 55(2006), p.453-456

[5] H.Zhong, Z.M.Ren, C.J.Li, Y.B.Zhong, W.D.Xuan and Q.L.Wang: Acta Metallurgica Sinica Vol. 51(2015), p.473-482(In Chinese)

[6] S.L.Tan, Q.Zhou, H.Wan: Special Casting \& Nonferrous Alloys Vol. 34(2014), p.1207-1210(In Chinese)

[7] G.M.Li, Y.Liu, X.Z.Zou, F.L.Yu, C.R.Li, E.G.Wang and K.Han: Journal of Functional Materials Vol. 44(2013), p.2197-2201(In Chinese)

[8] T.Zhang, W.L,Ren , J.W.Dong, X.Li, Z.M.Ren, G.H.Cao, Y.B.Zhong, K.Deng, Z.S.Lei and J.T.Guo: Journal of Alloys \& Compounds Vol. 487(2009), p.612-617

[9] Y.Y.Khine, J.S.Walker, F.R.Szofran: Journal of Crystal Growth Vol. 212(2000), p.584-596

[10] C.Y.Ban, L.Liu, D.D.Jiang, Q.X.Ba and J.Z.Cui: Transactions of Materials and Heat Treatment Vol. 32(2011), p.13-16(In Chinese)

[11] C.Y.Ban, D.D.Chen, Y.Han, Q.X.Ba and J.Z.Cui: Acta Metallurgica Sinica Vol. 44(2008), p.1224-1230(In Chinese)

[12] Z.H.Chen, W.J.Xia, Y.Q.Cheng: The Chinese Journal of Nonferrous Metals Vol. 15(2005), p.11(In Chinese) 